\title{
Angle-resolved measurements of the photoelectron spin polarization in the photoionization of $\mathrm{HI}$ molecules
}

\author{
N Böwering, M Müllert, M Salzmann and U Heinzmann \\ Universität Bielefeld, Fakultät für Physik, D-4800 Bielefeld, Federal Republic of Germany \\ and \\ Fritz-Haber-Institut der Max-Planck-Gesellschaft, D-1000 Berlin 33, Federal Republic of \\ Germany
}

Received 31 May 1991

\begin{abstract}
Using circularly polarized synchrotron radiation, the $5 \mathrm{p} \pi$ ionization of $\mathrm{HI}$ molecules is studied by means of angle-resolved photoelectron spin polarization spectroscopy. The energy dependences of the dynamical photoionization parameters are obtained and compared with an $a b$ initio calculation of Raseev $e$ t $a$ l and the corresponding data for the xenon atom. Angular distributions of the polarization component $A(\Theta)$ yield complete parameter sets. A strong influence of electronic autoionization is observed and analysed in terms of partial contributions using the additional information contained in the polarization results.
\end{abstract}

A complete determination of the dynamical photoionization parameters $\sigma, \beta, A, \xi$ and $\alpha$ requires angle-resolved photoelectron spectroscopy with analysis of the spin polarization components using circularly polarized radiation (Kessler 1985). For free atoms the first experiments of this type were performed for $5 \mathrm{p}$ ionization of xenon (Heckenkamp et al 1984) and permitted the extraction of the dipole transition matrix elements and phaseshift differences involved (Heckenkamp et al 1986b). For molecules, due to the non-spherical molecular field, the symmetry is decreased and many, in principle infinitely many, transitions can contribute. In addition, due to the averaging over all possible orientations of the molecular axis with respect to the photon momentum, the maximal degree of total photoelectron polarization is reduced to $50 \%$, while for atoms it can reach $100 \%$ (Cherepkov 1981). A general non-relativistic theory of the electron spin polarization for the case of molecules was given by Cherepkov (1981) and later extended and applied to the hydrogen halides by Raseev et al (1987).

First measurements demonstrating the polarization of molecular photoelectrons were performed for $\mathrm{CO}_{2}, \mathrm{~N}_{2} \mathrm{O}$ and $\mathrm{CH}_{3} \mathrm{Br}$ (Heinzmann et al 1980, 1981) near the ionization threshold in the spin-orbit autoionization region with angle-integrated detection using circularly polarized synchrotron radiation. The polarization parameter $\xi$ was also determined at several photon energies for heavy halogen and methyl halide molecules with unpolarized light (Schönhense et al 1984). More recently, a theoretical calculation including the influence of autoionization was compared with the experimentally determined wavelength dependence of the polarization parameter $\boldsymbol{A}$ for $\mathrm{HBr}$ molecules (Lefebvre-Brion et al 1989). Very recently, high-resolution angle-integrated polarization measurements were performed for $\mathrm{HI}$ resolving rotational structure (HuthFehre et al 1990).

† Present address: Bosch-Siemens HG, D-1000 Berlin 20, Federal Republic of Germany. 
In order to determine the photoelectron polarization which arises as a consequence of the spin-orbit interaction, it is essential to resolve the spin-orbit splitting. Therefore, techniques using angle-integrated polarization detection are restricted to the nearthreshold region where only one spin-orbit channel is open. For molecules containing heavy atoms, however, the spin-orbit splitting can be readily resolved by an electron spectrometer. With circularly polarized synchrotron radiation, all polarization parameters can then be studied over an extended energy region. It is the purpose of this paper to report on such studies for the relative partial cross sections $\sigma$ and the polarization parameters $A, \xi$ and $\alpha$ for photoelectrons corresponding to the two ${ }^{2} \Pi$ fine-structure final ionic states of $\mathrm{HI}^{+}(5 \mathrm{p} \pi)^{3}$ and also to present first results on the determination of all dynamical photoionization parameters at selected wavelengths obtained by angle- and spin-resolved spectroscopy. The HI molecule was chosen since it is isoelectronic to the xenon atom and comparisons in electronic excitation can be made due to the atomic-like character of the $(5 \mathrm{p} \pi)$ lone-pair orbital located at the iodine centre, and also since a theoretical prediction based on $a b$ initio calculations (Raseev et al 1987) is available. Furthermore, measurements of partial cross sections and the angular distribution parameters were reported for $\mathrm{HI}$ at photon energies above $16 \mathrm{eV}$ (Carlson et al 1984).

A description of the apparatus and the techniques employed in our experiment to obtain the data has been given previously (Heckenkamp et al 1986b). In brief, circularly polarized synchrotron radiation emitted out of plane at the storage ring BESSY at the $6.5 \mathrm{~m}$ normal-incidence monochromator (Schäfers et al 1986) was used for photoionization. At the exit focus, the target gas HI (better than $98 \%$ purity, supplied by Merck-Schuchardt) is introduced in an effusive molecular beam at room temperature. Behind the ionization region, a cold trap filled with liquid nitrogen is mounted and used to freeze out the gas; the background pressure inside the vacuum chamber was less than $9 \times 10^{-5} \mathrm{mbar}$. The photoelectrons emitted are energy analysed angle resolved using a hemispherical spectrometer which can be rotated in the reaction plane (see Heckenkamp et al 1986a). Two mutually perpendicular transverse polarization components are measured simultaneously in a subsequent high-energy Mott detector (acceleration voltage $100 \mathrm{kV}$, Sherman function -0.25 ). The reaction geometry and the definition of the spin polarization parameters determined are identical to the case of atomic photoionization described previously (Heckenkamp et al 1986b).

A typical photoelectron spectrum recorded at the magic angle $\left(\Theta_{m}=54.7^{\circ}\right)$ at a wavelength of $70 \mathrm{~nm}$ is shown in figure 1. It is similar to spectra obtained by other authors at higher photon energies (Lempka et al 1968, Turner et al 1970, Carlson et al 1984). Emission of an electron from the $(5 \mathrm{p} \pi)$ orbital of $\mathrm{HI}$ gives rise to the spin-orbit-split ionic ground states, $\mathrm{HI}^{+} \mathrm{X}^{2} \Pi_{3 / 2}(v=0)$ and $\mathrm{X}^{2} \Pi_{1 / 2}(v=0)$, at binding energies of $10.38 \mathrm{eV}$ and $11.05 \mathrm{eV}$, respectively. Creation of a $(5 \mathrm{p} \sigma)$ hole leads to the $\mathrm{HI}^{+} \mathrm{A}^{2} \Sigma_{1 / 2}^{+}$excited state in several vibrational levels which are, however, not distinctly observed even at higher resolution because of strong broadening caused by predissociation. Due to the non-bonding character of the $(5 \mathrm{p} \pi)$ orbital the Franck-Condon overlap is such that the ${ }^{2} \Pi$ states are almost exclusively populated in the vibrational ground state unless the influence of autoionization resonances is strong (see also Böwering et al 1991).

In figure 2 we show partial photoionization cross sections in the region of $120 \mathrm{~nm}$ $60 \mathrm{~nm}$ for the two final ionic states ${ }^{2} \Pi_{3 / 2}(v=0)$ and ${ }^{2} \Pi_{1 / 2}(v=0)$. Relative cross section data were obtained from intensity measurements using a channeltron as detector and the spectrometer at $\Theta_{\mathrm{m}}$ in the constant ionic state (cIs) mode while the monochromator 


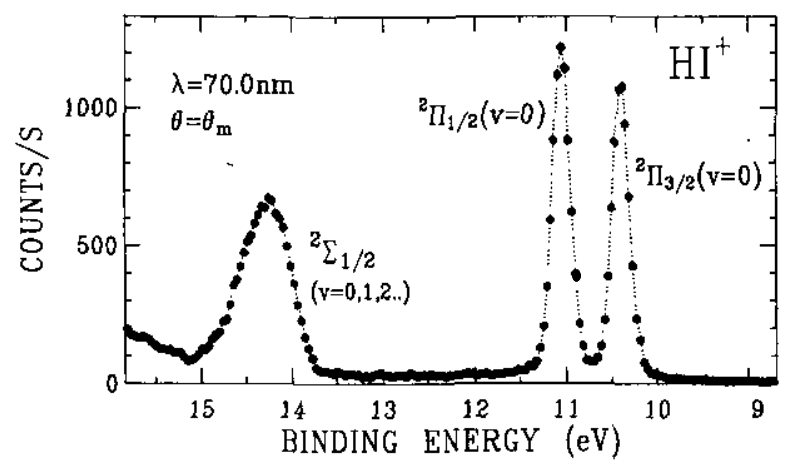

Figure 1. Typical photoelectron spectrum of $\mathrm{HI}$ obtained at the magic angle $\Theta_{\mathrm{m}}$ at a photon wavelength of $70 \mathrm{~nm}$.
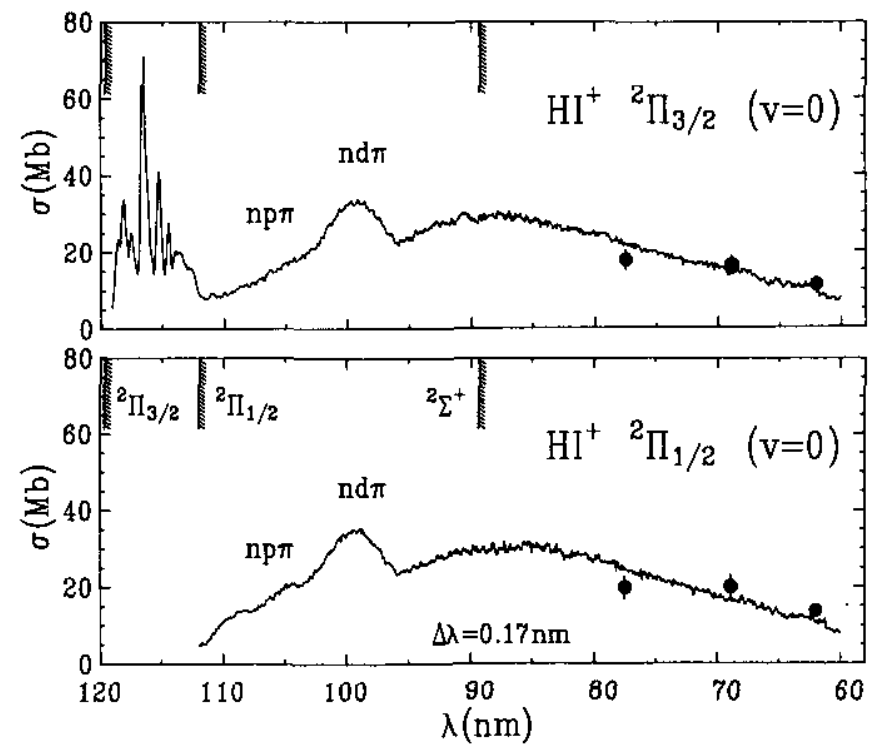

Figure 2. Partial photoionization cross sections for the final ionic states $\mathrm{HI}^{+2} \Pi_{3 / 2}(v=0)$ and ${ }^{2} \Pi_{1 / 2}(v=0)$ at a spectral resolution of $0.17 \mathrm{~nm}$. As explained in the text, the relative intensity results were matched to the absolute data of Carlson et al (1984) which are shown for comparison (full circles). The relevant ionization limits are indicated by vertical lines.

was operated with a 3600 lines $/ \mathrm{mm}$ grating $(\Delta \lambda=0.17 \mathrm{~nm})$. Corrections were made for changes in target gas pressure and also for the reduction in the radiation intensity due to the decrease in current of the beam in the storage ring. In addition, the spectra were corrected for the combined effect of the wavelength dependence of the photon intensity at the exit focus of the monochromator and the transmission characteristics of the electron spectrometer. This was achieved by recording cis runs with identical set-up for the $\mathrm{Xe}^{+2} \mathrm{P}_{3 / 2}$ state for which the cross section is known precisely (West and Morton 1978). In this way, relative partial cross section data $\nmid$ were obtained for the

$\dagger$ Photoelectrons with kinetic energies below $0.15 \mathrm{eV}$ are not collected efficiently in our set-up. Therefore the cross sections are inaccurate near threshold. 
$\mathrm{HI}^{+2} \mathrm{II}$ final ionic states and then matched with the data reported by Carlson et al (1984) (also plotted in the figure) to yield an absolute scale.

In the wavelength region between $119.4 \mathrm{~nm}$ and $112.2 \mathrm{~nm}$ the photoionization to $\mathrm{HI}^{+2} \Pi_{3 / 2}(v=0)$ is dominated by spin-orbit autoionization of Rydberg states converging towards the ${ }^{2} \Pi_{1 / 2}(v=0)$ state (Eland and Berkowitz 1977, Lefebvre-Brion et al 1985, Hart and Hepburn 1989). Due to the spectral bandwidth of $0.17 \mathrm{~nm}$ the resonance structure is only incompletely resolved. The absolute cross section scale for the spinorbit autoionization region obtained by the procedure outlined above does also agree fairly well with the data of Carlson et al (1986) given for this region.

Above the ${ }^{2} \Pi$ thresholds, a broad resonance structure centred near $100 \mathrm{~nm}$ is observed for both ionic channels in agreement with total cross section data (Terwillinger and Smith 1975, Dehmer and Chupka 1978) which is attributed to electronic autoionization of predissociating Rydberg states converging to $\mathrm{HI}^{+} \mathrm{A}^{2} \Sigma^{+}$. In analogy to $\mathrm{HBr}$ (Lefebvre-Brion et al 1989) it is assigned to $(\mathrm{d} \pi)$ resonances with effective principal quantum number $n^{*}=2.78$ (Terwillinger and Smith 1975). Likewise, the region around $110 \mathrm{~nm}$ corresponds to $(n \mathrm{p} \pi)$ Rydberg states with ${ }^{2} \Sigma^{+}$core. Partial cross section data have not been reported before for this region. In the range from threshold to $\lambda=90 \mathrm{~nm}$, there is also significant population of vibrationally excited open ionization channels (Böwering et al 1991). Another weak maximum of the cross sections is reached near the $\mathrm{A}^{2} \Sigma_{1 / 2}^{+}$threshold in a wavelength region where also the yield of $\mathrm{I}^{+}$ions increases (Eland and Berkowitz 1977). Throughout the range examined here the branching ratio $\sigma\left({ }^{2} \Pi_{3 / 2}\right) / \sigma\left({ }^{2} \Pi_{1 / 2}\right)$ at equal photon energy is almost constant with a value of about 0.9 .

Spin polarization measurements were performed operating the monochromator with the 1200 lines $/ \mathrm{mm}$ grating in first order $(\Delta \lambda=0.5 \mathrm{~nm})$ for intensity reasons. The parameters $A$ (characterizing the component $A(\Theta)$ of the spin polarization vector parallel to the photon momentum) and $\xi$ (describing the component $P_{\perp}(\Theta)$ perpendicular to the reaction plane) were determined using the Mott detector in a measurement cycle with the spectrometer at the angles $+\Theta_{m}$ and $-\Theta_{m}$ with both left- and right-handed circularly polarized light (degree of circular polarization: $92 \%$ ). The results for the spin polarization parameters $A, \xi$ and $\alpha$ obtained for the final ionic states $\mathrm{HI}^{+2} \Pi_{3 / 2}$ $(v=0)$ and ${ }^{2} \Pi_{1 / 2}(v=0)$ are given in figure 3 (open and full circles, respectively). The full curves represent the $a b$ initio calculation of Raseev et al (1987) $\dagger$. These first theoretical results for molecules which provide all spin polarization parameters were obtained in a frozen-core static-exchange approximation by a non-relativistic calculation for the open continuum.

The experimental data for the parameters $A, \xi$ and $\alpha$ each show opposite signs for the two spin-orbit components ${ }^{2} \Pi_{3 / 2}$ and ${ }^{2} \Pi_{1 / 2}$. This reveals directly the influence of the spin-orbit interaction of the ${ }^{2} \Pi$ final ionic state. Since the two substates have equal statistical weight, the corresponding polarization parameters should have equal magnitude and opposite signs (at equal photoelectron energy) within the non-relativistic model (Cherepkov 1981). Deviations from this equality reflect the influence of the spin-orbit interaction in the continuum states.

As also seen from the cross section results of figure 2 , the data can be divided into two regimes: a region below the ${ }^{2} \Sigma^{+}$limit which is strongly influenced by resonances and the region of the unperturbed open continuum at higher photon energies. Spin polarization data were first taken at large intervals to cover the accessible wavelength region. In order to examine the influence of the electronic autoionization resonances

$\dagger$ Raseev et al (1987) use the notation $\vec{P}, \xi$ and $\gamma$ for the spin polarization parameters. 


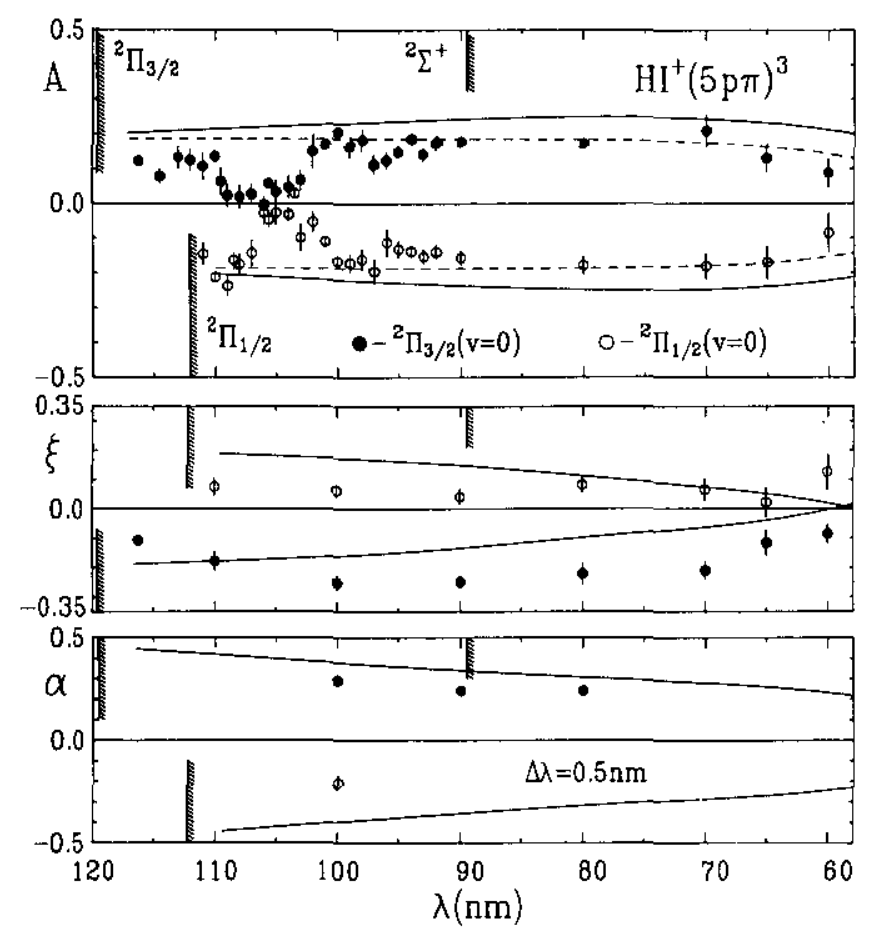

Figure 3. Wavelength dependence for the three polarization parameters $A, \xi$ and $\alpha$ for $\mathrm{HI}^{+2} \Pi_{3 / 2}(v=0)$ (full circles) and $\mathrm{HI}^{+2} \Pi_{1 / 2}(v=0)$ (open circles) final ionic states from $120 \mathrm{~nm}$ to $60 \mathrm{~nm}$ (spectral resolution: $0.5 \mathrm{~nm}$ ). The full curves represent the non-relativistic $a b$ initio calculation of Raseev et al (1987). The broken curves for the parameter $A$ indicate the scaled xenon data of Heckenkamp et al (1986b).

in more detail, however, the parameter $A$ was measured with much higher point density in the range between 112 and $90 \mathrm{~nm}$. In this region strong deviations from the theoretical prediction (which does not take autoionization into account) exist. At around $105 \mathrm{~nm}$ $\boldsymbol{A}$ approaches zero for both substates; i.e. the total polarization transfer from photon to photoelectron becomes vanishingly small at this photon wavelength. Nevertheless, the non-relativistic relationship for $A$ is not only fulfilled to a very good degree for the open continuum, but also for the resonance region of electronic autoionization (where the comparison has to be made at equal photon energies). A deviation occurs only near $108 \mathrm{~nm}$. In comparison to the theoretical curve for the open continuum region the wavelength dependence for the $A$ parameters measured is similar but the absolute values are smaller.

For the region of spin-orbit autoionization between the ${ }^{2} \Pi$ limits the parameter $A$ was measured only in the peak of the $n=6$ Rydberg member and at two further wavelength positions $\left({ }^{2} \Pi_{3 / 2}\right.$ final ionic state), since much higher resolution is required for an investigation of this region. However, the values obtained are consistent with the high-resolution results (Huth-Fehre et al 1990). For the region of electronic autoionization, on the other hand, the resolution of the experiment is adequate, since these structures are strongly broadened due to predissociation.

While the values for the parameter $\boldsymbol{A}$ show the same tendency as the calculation. in the range of $90 \mathrm{~nm}$ to $60 \mathrm{~nm}$, a striking deviation is observed for the parameter $\xi$ from both the theoretical prediction as well as from the non-relativistic relationship. 
Only the coarse structure was measured for this polarization parameter; between $120 \mathrm{~nm}$ and $90 \mathrm{~nm}$ resonance features due to autoionization cannot be definitely excluded based on our results. We have also obtained $A=(-0.085 \pm 0.02)$ and $\xi=(-0.01 \pm 0.02)$ at $\lambda=70 \mathrm{~nm}$ for the ${ }^{2} \Sigma_{1 / 2}^{+}$final ionic state. The non-zero value for $A$ shows that relativistic effects are not negligible; the value for $\xi$ could suggest that the theoretical description in Hund's coupling case (a) (Raseev et al 1987) is adequate.

By rotating the spectrometer in the reaction plane, first values were also obtained for the spin polarization asymmetry parameter $\alpha$ describing the angular distribution of the polarization component $A(\Theta)$. The angular distributions $A(\Theta)$ measured at $\lambda=100 \mathrm{~nm}$ (angular resolution $\Delta \Theta=5^{\circ}$ ) are plotted in figure 4 for the two final ionic states. The full curve represents a non-linear least squares fit according to the angular distribution in our reaction geometry,

$$
A(\Theta)=\frac{A-\alpha P_{2}(\cos \Theta)}{1-(\beta / 2) \bar{P}_{2}(\cos \Theta)}
$$

where $P_{2}(\cos \Theta)$ is the second Legendre polynomial (Heckenkamp et al 1984). The fit yields the parameters $A, \alpha$ and $\beta \dagger$ and also confirms the analytic form of equation (1) for the case of photoionization of molecules. Since the complete angular distribution has to be recorded only a few points were taken for $\alpha$. At these positions the fit parameters together with $\sigma$ and $\xi$ yield a complete set of dynamical parameters. The $\alpha$-values for the ${ }^{2} \Pi_{3 / 2}$ state show the same tendency as the calculated values but are smaller in magnitude. Typically, in contrast to the atomic case, for randomly oriented free molecules the angular distribution is less pronounced due to the averaging over all orientations of the molecular axis (see also Cherepkov 1981). The distributions for $\mathrm{HI}$ are of opposite shape for the two ${ }^{2} \Pi$ spin-orbit components with a maximum for $A(\Theta)$ for the ${ }^{2} \Pi_{1 / 2}$ state at $\Theta=0^{\circ}$ in correspondence to the (more pronounced) distributions for the ${ }^{2} \mathrm{P}_{3 / 2}$ and ${ }^{2} \mathrm{P}_{1 / 2}$ ionic states of the xenon atom (Heckenkamp et al 1984).
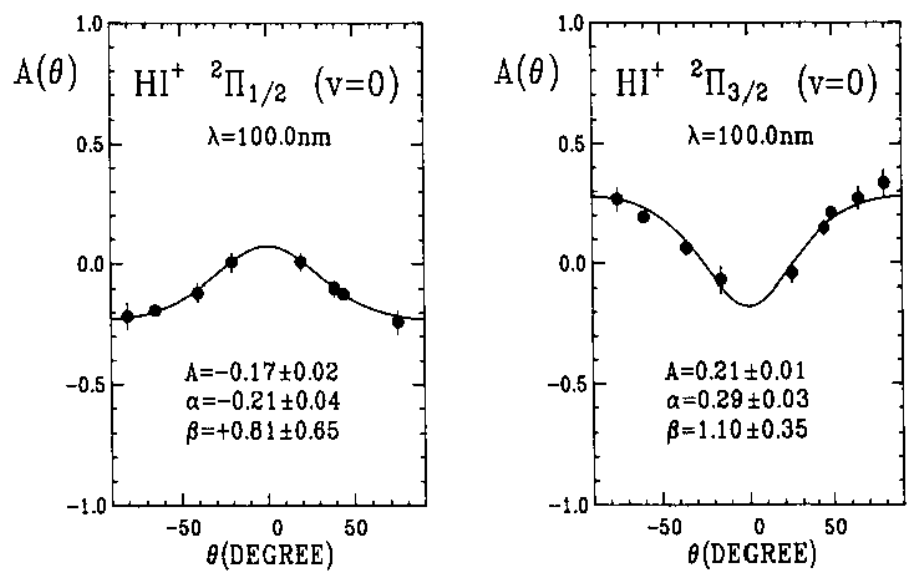

Figure 4. Experimental data points (full circles) and fit results (full-curves) for the angular distribution of the polarization component $A(\Theta)$ for $\mathrm{HI}^{+2} \Pi_{3 / 2}(v=0)$ and ${ }^{2} \Pi_{1 / 2}(v=0)$ at $\lambda=100 \mathrm{~nm}$. 
In order to discuss the strength of the different channels the results for the phase-independent parameters $\sigma$ and $A$ can be combined to analyse the various contributions to the photoemission in terms of partial cross sections $\sigma_{\lambda}(\lambda=\sigma, \pi, \delta)$ within the non-relativistic formalism. One has:

$$
A= \pm \frac{1}{2}\left(\sigma_{\delta}-\sigma_{\sigma}\right) / \sigma_{\text {tot }}
$$

(Raseev et al 1987, equation (31)), where the plus sign refers to the ${ }^{2} \Pi_{3 / 2}$ final state $\left(A_{3 / 2}\right)$ and the minus sign to ${ }^{2} \Pi_{1 / 2}\left(A_{1 / 2}\right)$ and

$$
\sigma_{\mathrm{tot}}=\sigma_{\sigma}+\sigma_{\pi}+\sigma_{\delta}
$$

is the total cross section. The limiting values $A= \pm 0.5$ occur when there are only $\sigma_{\delta}$ or $\sigma_{\sigma}$ contributions, respectively, and $A=0$ implies that $\sigma_{\delta}=\sigma_{\sigma}$. The fact that $A_{3 / 2}$ is positive throughout the range examined shows that the $\delta$ contributions are larger than the $\sigma$ contributions. This is in analogy to the isoelectronic xenon atom where the $\mathrm{d}$ continuum dominates. In fact, taking atomic-like transition moments (thus considering only the $n \mathrm{p} \pi \rightarrow \varepsilon \mathrm{d} \lambda$ transitions), one obtains $A_{3 / 2}=0.25$ (Raseev et al 1987); the data thus indicate that the $\varepsilon \mathrm{d} \delta, \varepsilon \mathrm{d} \pi$ waves are the main ionization channels.

By comparing figures 2 and 3, one observes that almost all structures present in the cross section data are reflected by corresponding features in the polarization parameter $A$. In particular, the broad maximum at around $\lambda=100 \mathrm{~nm}$ and the distinct sharp minimum at $\lambda=96 \mathrm{~nm}$ can also be seen in the data for $A$. From comparison with $\mathrm{HBr}$ (Lefebvre-Brion et al 1989), the most intense resonances should be ${ }^{1} \Pi$ states with $\mathrm{A}^{2} \Sigma^{+}$core and $(n \mathrm{p} \pi)$ and $(n \mathrm{~d} \pi)$ Rydberg configuration. They can autoionize only into the ${ }^{2} \Pi \varepsilon \sigma$ or $\varepsilon \delta$ continua and should not give rise to $\varepsilon \pi$ outgoing waves. For the $(\mathrm{d} \pi)$ resonance region the polarization values increase in magnitude indicating that the $\varepsilon \mathrm{d} \delta$ waves dominate (partial waves with $(l=2)$ should be most intense for $\varepsilon \delta$ ). On the other hand, in the wavelength range from $102 \mathrm{~nm}$ to $108 \mathrm{~nm}$ (mainly (p $\pi$ ) resonances) the $\varepsilon \sigma$ waves are almost as strong as the $\varepsilon \delta$ waves. In the region from threshold to $108 \mathrm{~nm}$ the $\varepsilon \delta$ waves are again more intense than the $\varepsilon \sigma$ waves.

The calculation of Raseev et al (1987) predicts that the parameter $A$ should reach 0.25 near $\lambda=80 \mathrm{~nm}$ which is not observed. However, the experimental values for $\mathrm{HI}$ can also be compared with the $A$-values for xenon where theories using random phase approximation are in close agreement with the corresponding experimental data (Heckenkamp et al 1986b). Therefore, the results for Xe are shown in figure 4 as broken curves. For proper comparison at equal photoelectron energy the Xe results were scaled to the different ionization limits of $\mathrm{HI}$ and weighted appropriately. Remarkably, the agreement is more favourable for the xenon data than for the one-configuration calculation for $\mathrm{HI}$. This indicates on the one hand that correlations play a significant role and also that the photoionization of $\mathrm{HI}(5 \mathrm{p} \pi)^{4}$ shows strikingly strong atomic-like character in the continuum region. Similar conclusions hold for $\alpha$ but not for $\xi$ which has a complicated phase dependence as discussed below.

In order to examine the validity of a non-relativistic description of the $n \mathrm{p} \pi$ photoionization process for $\mathrm{HI}$ one can form the ratios of the dynamical parameters for the two ${ }^{2} \Pi$ substates. As already pointed out above, the cross section branching ratio is nearly constant and the non-relativistic model (Cherepkov 1981) is also fulfilled for the parameter $A$. As noted by Lefebvre-Brion et al (1986), at higher photon energies this holds also for the parameter $\beta$ (Carlson et al 1984). However, the data for the parameter $\xi$ deviate strongly from the non-relativistic relationship. Similar observations in experiments for the parameter $\xi$ were made before for molecules containing heavy 
halogen atoms (Schönhense et al 1984). $\xi$ shows the most important phase dependence since off-diagonal interference terms contribute most strongly in the theory (Raseev et al 1987) and its dynamical factors depend on the sine of phaseshift differences of continuum wavefunctions. (The parameters $\beta$ and $\alpha$ have a cosine-like phase dependence and are dominated by diagonal terms.) Therefore $\xi$ is the most sensitive parameter to anisotropic interactions like the spin-orbit interaction in the continuum states.

In conclusion, we have presented the first angle-resolved measurements to determine all dynamical molecular photoionization parameters and to verify the angular distribution of the polarization component $A(\Theta)$ for molecules using $\mathrm{HI}$ as a sample gas. For the open continuum region, the comparison with the $a b$ initio calculation of Raseev et al (1987) shows the same tendency for the energy dependence of the dynamical parameters with the exception of the phase-sensitive parameter $\xi$. The results for $\mathrm{HI}$ show striking similarities to the corresponding data for the xenon atom. A strong influence of electronic autoionization resonances was observed for the partial cross sections $\sigma$ and the total polarization transfer $A$ which can be combined to yield additional information on the strengths of the outgoing partial waves. Since complete sets of dynamical parameters can be obtained, this experiment opens the way for detailed studies of the dominant contributions to the outgoing partial waves in molecular photoionization.

\section{Acknowledgments}

The authors would like to thank H-W Klausing, R Kuntze, H Lefebvre-Brion and G Raseev for useful discussions. The co-operation of the staff at BESSY is gratefully acknowledged. This work was supported by BMFT under contract 05431 AX/TP2 and the European Commission.

\section{References}

Böwering N, Klausing H.W, Müller M, Salzmann M and Heinzmann U 1991 Chem. Phys. Lett. in press Carlson T A, Fahlmann A, Krause M O, Keller P R, Taylor J W, Whitley T and Grimm F A 1984 J. Chem. Phys. 80 3521-7

Carlson T A, Gerard P, Krause M O, Von Wald G, Taylor J W and Grimm F A 1986 J. Chem. Phys. 84 $4755-9$ Cherepkov N A 1981 J. Phys. B: At. Mol. Phys. $142165-77$

Dehmer P M and Chupka W A 1978 Argonne National Laboratory Report ANL-78-65 part I, pp 13-18

Eland J H D and Berkowitz J 1977 J. Chem. Phys. 67 5034-9

Hart D J and Hepburn J W 1989 Chem. Phys. 129 51-64

Heckenkamp Ch, Eyers A, Schäfers F, Schönhense G and Heinzmann U 1986a Nuci. Instrum. Methods A 246 500-3

Heckenkamp Ch, Schäfers F, Schönhense G and Heinzmann U 1984 Phys. Rev. Lett. 52 421-4

1986b Z. Phys. D 2 257-74

Heinzmann U, Osterheld B, Schäfers F and Schönhense G 1981 J. Phys. B: At. Mol. Phys. 14 L79-84

Heinzmann U, Schäfers F and Hess B A 1980 Chem. Phys. Lett. 69 284-9

Huth-Fehre T, Mank A, Drescher M, Böwering N and Heinzmann U 1990 Phys. Rev. Lett. 64 396-9

Kessler J 1985 Polarized Electrons 2nd edn (Berlin: Springer)

Lefebvre-Brion H, Giusti-Suzor A and Raseev G 1985 J. Chem. Phys. 83 1557-66

Lefebvre-Brion H, Raseev G and Le Rouzo H 1986 Chem. Phys. Lett. 123 341-4

Lefebvre-Brion H, Salzmann M, Klausing H W, Müller M, Böwering N and Heinzmann U $1989 \mathrm{~J}$. Phys. B: At. Mol. Opt. Phys. 22 3891-900

Lempka H J, Passmore T R and Price W C 1968 Proc. R. Soc. A 304 53-64 
Raseev G, Keller F and Lefebvre-Brion H 1987 Phys. Rev. A 36 4759-74

Schäfers F, Peatman W, Eyers A, Heckenkamp Ch, Schönhense G and Heinzmann U 1986 Rev. Sci. Instrum. $571032-41$

Schönhense G, Dzidzonou V, Kaesdorf S and Heinzmann U 1984 Phys. Rev. Lett. 52 811-4

Terwillinger T D and Smith A L $1975 \mathrm{~J}$. Chem. Phys, 63 1008-20

Turner D W, Baker C, Baker A D and Brundle C R 1970 Molecular Photoelectron Spectroscopy (New York: Wiley) p 59

West J B and Morton J 1978 At. Data Nucl. Data Tables 22 103-7 